\title{
Perinatal Transmission of Dengue: A Case Report
}

\author{
Navjot Kaur ${ }^{1}$, Dhananjay K Mangal², Sushil Gupta ${ }^{3}$, Chanchal Singhal ${ }^{4}$, Gajinder Pal Singh Kaler ${ }^{5}$ \\ 1, 2, 3 Department of Neonatology, Babylon Hospital, Jaipur, Rajasthan, India ${ }^{4}$ Department of Paediatrics, Babylon \\ Hospital, Jaipur, Rajasthan, India. ${ }^{5}$ Department of Cardiology, SMS Hospital, Jaipur, Rajasthan, India.
}

\section{PRESENTATION OF CASE}

A single, male neonate was born at 36 weeks of gestation with birth weight of 2.62 $\mathrm{Kg}$, to a 24-year-old primigravida mother by LSCS in an outside hospital. Apgar score at $1 \mathrm{~min}$ and 5 mins were 7 and 9 respectively and he was shifted to mother's side. On day 4 of life, he developed respiratory distress and decreased oral intake and was referred to our neonatal tertiary care unit (NICU).

Maternal antenatal history was uneventful. Mother had fever and thrombocytopenia and was diagnosed as dengue infection (Dengue NS 1 antigen and Ig M positive) 3 days before delivery and managed at same hospital.

On admission, baby had respiratory rate of 64 / min, mild retractions, Sp02 of 92 $\%$ on room air and mild hepatomegaly. Cardiovascular system seemed to be normal but poor activity and sucking was there. He was started on IV fluids, antibiotics and non-invasive respiratory support. Chest X-ray was normal. Haemoglobin was $18.3 \mathrm{gm}$ / dL, total leukocyte count of $4600 / \mathrm{mm}^{3}$, absolute neutrophil count of 2681, platelet count of 1.5 lac, haematocrit of $50.8 \%$ and C reactive protein of 7.7. Dengue NS1 antigen and IgM came out to be positive. There was no evidence of mosquito bite in neonate postnatally.

Infant's clinical condition deteriorated with increased requirement of respiratory support and SpO2 up to $40 \%$. Platelet count further fell to 59000 on day 2 and 13000 on day 3 of hospital stay and was managed with multiple platelet transfusions. Baby also had features of third space loss. Repeat chest X-ray suggestive of right pleural effusion and ultrasound abdomen showed mild ascites and mild hepatomegaly. But cranial ultrasound was also done which was normal. No evidence of bleeding during hospital stay. Baby was managed initially with fluids and first line antibiotics as per the unit protocol. Orogastric feeds were started as baby had clinical improvement which were built up gradually and baby switched to spoon feeds after 7 days. Baby was weaned off from respiratory support as requirement decreased. Baby was discharged with improving platelet count and absence of features of third space loss. On discharge baby was hemodynamically stable and accepting oral feed.
Corresponding Author: Dr. Navjot Kaur. Department of Neonatology, Babylon Hospital, Jaipur, Rajasthan, India. E-mail: cutiesweetiepiez@gmail.com

DOI: $10.14260 / j e m d s / 2020 / 693$

How to Cite This Article:

Kaur N, Mangal DK, Gupta S, et al. Perinatal transmission of dengue. J Evolution Med Dent Sci 2020;9(42):3167-3168, DOI: 10.14260/jemds/2020/693

Submission 06-07-2020,

Peer Review 07-09-2020,

Acceptance 14-09-2020,

Published 19-10-2020.

Copyright (C) 2020 Navjot Kaur et al. This is an open access article distributed under Creative Commons Attribution License [Attribution 4.0 International (CC BY 4.0)] 
Dengue is a systemic arthropod borne and transmitted systemic viral infection which is an emerging disease in many states of India. Major mode of transmission of dengue is mosquito bite but vertical transmission is rarely seen. ${ }^{1}$ There are some reported cases of dengue infection in pregnancy and association with many complications in neonate like low birth weight, perinatal mortality, foetal distress and preterm birth. The samples from foetal or cord blood are considered as a better source of qualifying dengue infection in utero. ${ }^{2}$ In neonates, vertical transmission of dengue from mother can produce varying symptoms ranging from fever with thrombocytopenia to intracranial haemorrhage. ${ }^{3}$

As India is a resource limited country the survey for dengue cases in neonates is not done in a schematic manner, there are only a few case reports of neonatal dengue infection from India and its vertical transmission. Here we are reporting a case of vertical transmission of dengue in a neonate at our Neonatal ICU.

\section{DISCUSSION}

In India, transmission of dengue from mother to foetus is not so uncommon but it is often misdiagnosed leading to failure to report. This underreporting could also be due to asymptomatic infection and the symptoms mimic other viral infections. ${ }^{4}$ Majority of the reported neonatal dengue cases are from the mothers who got infected in later part of pregnancy as compared to early pregnancy. As less maternal antibodies pass to foetus, this exposes the foetus to a greater risk of infection with dengue. The unprotected new born is thus exposed to virus load from mother. ${ }^{5}$ The incubation period of dengue virus is 3 - 10 days in humans and in neonates its halflife is 40 days. 6,7

The mother of this baby had history of fever for 3 days prior to diagnosis of dengue so it got transmitted to neonate. A systematic review conducted by Sawyer et al stated that higher incidence of transmission of dengue in c section deliveries, premature births and preeclampsia. ${ }^{8}$ This finding is consistent with our case report. The factors involved in the pathogenesis of dengue in new-born are maternal age, time of pregnancy and gestational age. ${ }^{9}$ Maternal age of less than 20 years is suggested to be associated with higher amount of circulating protective anti-dengue antibodies as compared to mothers of older age. The severity of dengue infection has been found to be more in neonates with lower birth weight. ${ }^{10}$ In early pregnancy, during the period of organogenesis, this virus has ability to cause spontaneous abortions and alterations in the neural tube. In later pregnancy, the passage of virus through the placenta has serious implications in the neonate. The mechanism of thrombocytopenia involves platelet activation, pro-coagulant and anticoagulant pathway activation, various compliment factors, cytokines and endothelial cells. The destruction of platelets and thrombocytopenia are associated with dengue fever and it could be due to bone marrow suppression and the peripheral destruction of platelets induced by platelet antibodies. The role of platelets in the viral spread is not clear till now. There is need for regular platelet count monitoring. Any bleeding manifestations need to be looked for and intervention made in time. In our case, ultrasound cranium of the neonate was done and no evidence of intracranial haemorrhage was detected.

\section{CONCLUSIONS}

There is significant need for highlighting the perinatal transmission of dengue especially in endemic areas of the world like India. In order to reduce the neonatal mortality, prompt diagnosis of dengue has to be considered, and intervention initiated. Adequate hydration and appropriate laboratory investigations can prevent worsening. Vigilant monitoring is the key to uneventful recovery in a neonate.

Financial or other competing interests: None.

Disclosure forms provided by the authors are available with the full text of this article at jemds.com.

\section{REFERENCES}

[1] Barthel A, Gourinat AC, Cazorla C, et al. Breast milk as a possible route of vertical transmission of dengue virus? Clin Infect Dis 2013:57(3):415-7.

[2] Perret C, Chanthavanich P, Pengsaa K, et al. Dengue infection during pregnancy and transplacental antibody transfer in Thai mothers. J Infect 2005;51(4):287-93.

[3] Basurko C, Carles G, Youssef M, et al. Maternal and foetal consequences of dengue fever during pregnancy. Eur J Obstet Gynecol Reprod Biol. 2009;147(1):29-32.

[4] Grunnill M, Boots M. How important is vertical transmission of dengue viruses by mosquitoes (diptera: culicidae)? J Med Entomol 2016;53(1):1-19.

[5] Carroll ID, Toovey S, Van Gompel A. Dengue fever and pregnancy: a review and comment. Travel Med Infect Dis 2007;5(3):183-8.

[6] Chan M, Johansson MA. The incubation periods of dengue viruses. PLoS One 2012;7(11):e50972.

[7] Watanaveeradej V, Endy TP, Samakoses R, et al. Transplacentally transferred maternal-infant antibodies to dengue virus. Am J Trop Med Hyg 2003;69(2):123-38.

[8] Pouliot SH, Xiong X, Harville E, et al. Maternal dengue and pregnancy outcomes: a systematic review. Obstet Gynecol Surv 2010;65(2):107-18.

[9] Sirinavin S, Nuntnarumit P, Supapannachart S, et al. Vertical dengue infection: case reports and review. Pediatr Infect Dis J 2004;23(11):1042-7.

[10] Kerdpanich A, Watanaveeradej V, Samakoses R, et al. Perinatal dengue infection. Southeast Asian J Trop Med Public Health 2001;32(3):488-93. 